\title{
Why Online does not Equal Offline: Comparing On- line and Real-World Political Support Among Politicians.
}

\author{
Laurence Brandenberger ${ }^{(1)} \quad$ Giona Casiraghi $^{(1)}$ \\ Georges Andres ${ }^{(1)} \quad$ Simon Schweighofer ${ }^{(1,2)}$ \\ Frank Schweitzer ${ }^{(1)}$
}
(1) ETH Zurich, Switzerland
(2) Complexity Science Hub Vienna, Austria

\begin{abstract}
Are offline political relations and endorsements established or maintained with online interactions? We examine the external validity of data on political behavior exhibited online and compare it to offline behavior. We overlay two data sets on political support among members of the Swiss parliament to estimate the explanatory power of online endorsements on offline cosponsorship signatures among members, using a gHypEG network regression model. Whereas offline support behavior is driven by a broad range of factors, such as network and homophily effects, online endorsement is less diverse. Our findings show that online endorsement is predominantly driven by partisanship, calling into question the use of online political behavior data to study real-world politics.
\end{abstract}

Keywords: political support, online and offline behavior, legislative politics, cosponsorhip, legislative networks.

\footnotetext{
${ }^{*}$ Corresponding author at: Chair of Systems Design, ETH Zurich, Weinbergstr. 56/58, 8092 Zurich, Switzerland

E-mail address: lbrandenberger@ethz.ch Orcid ID: https : / / orcid.org/ 0000-0003-0392-9766 This work was supported by the Swiss National Science Foundation (Grant number 184963, 'Analyzing cosponsorship Networks from 127 Years of the Swiss Federal Assembly'). The authors would like to thank the participants of the Social Systems Session at NetSci 2019 and participants of the 12th Annual Political Networks Conference 2019 for helpful comments and discussions.
} 


\section{Introduction}

Data from online political interactions are more and more used to study and to explain realworld politics. This development is mainly driven by the availability of data from social media platforms (Grimmer 2015) and the increasing number of tools to model web-based data (e.g., Barberá 2015, Garcia et al., 2012, Beauchamp 2017).For instance, data from online social media platforms such as the microblogging platform Twitter have been used to study patterns in alliance formation among politicans (Cherepnalkoski and Mozetič 2016), political ideology (Bond and Messing 2015), mobilization (Alvarez et al. 2015), voting outcomes (DiGrazia et al. 2013) or issue attention (Barberá et al., 2019).

These studies are often generalized into real-world political behavior, presupposing that a clear-cut comparison between online and offline politics is justified. But to what extent can online political behavior be compared to offline behavior? How much can online behavior inform offline politics, and what can we learn from online political behavior?

We address these questions in our paper with a direct comparison of online and offline political support among politicians, specifically members of the Swiss parliament (MPs). For this comparison we use data about their cosponsorship support in parliament and their endorsement clicks on the Swiss social media platform Politnetz.

Online political endorsement can be seen as a positive exchange among politicians on social media portals, leaving positive comments on another politician's post or tagging collaboration partners in social media appearances. We analyze data from the online networking and debate platform Politnetz, which was founded in 2009 to provide politicians and the general public with forum for political discussions and to foster transparency in politics. The portal was widely used until 2016 and includes over 3,000 politicians (local, cantonal/state and national level) (Garcia et al. 2015). Apart from discussing political issues, users of Politnet $z$ were given a chance to openly demonstrate their support for other politicians by clicking the endorsement button on the politician's profile page. Users also present their list of supportees on their own profile page. This endorsement button can be compared to following another politician on Twitter.

Offline political support comes in many forms, such as praising another politician in a speech, exchanging information, collaborating together on a project or hosting events together. Our focus is on cosponsorship as a widely studied signature of political support among MPs (e.g., Campbell, 1982, Kessler and Krehbiel, 1996, Fowler, 2006: Bratton and Rouse, 2011). We analyze 
data from two legislative periods (2007-2015) of the Swiss parliament. During that period 312 different MPs were present and more than 91,000 cosponsorship events of legislative proposals or bills ${ }^{1}$ were recorded.

MPs co-sign bills to show their preferences for a particular political issue. Apart from internal signaling, we also know that cosponsorship support mirrors a rich set of personal relations among MPs. Alternative motivations to cosponsor a proposal are personal ties (e.g., friendship or familial) among the sponsor and cosponsor of a bill, notions of reciprocity or favor trading (Brandenberger 2018), coalition formation or group dynamics, such as homophily or party cohesion (Craig et al. 2015, Bratton and Rouse, 2011, Tam Cho and Fowler, 2010). Such insights give us important information for developing our model. Obviously, cosponsorship signatures depend on another, thus we cannot analyze them the same way as we would analyze independent observations. These studies also motivate that reciprocity, homophily and party membership should be considered as control variables later in our model.

To operationalize cosponsorship support among MPs, we have chosen a network representation. Each node in this network represents an MP and directed edges between MPs represent (repeated) cosponsorship support from one MP to another. This results in a multi-edge network $\mathcal{G}$, where edge counts range form zero (no support between two MPs) to the maximum number of bills an MP proposed to parliament. For instance, if MP a proposed 12 bills, other MPs $i$ can support a 0 -12 times.

The existence of repeated interactions is often left out in the analysis of cosponsorship networks (e.g., Fischer et al. 2019). Instead, the network $\mathcal{G}$ is simplified to a binary version which only distinguishes whether or not two MPs have supported another, but not how much. This introduces a bias because one-time support counts the same as repeated support over a long time. To avoid such biases, we analyze the multi-edge network as is.

To compare online and offline political support among MPs, we use a gHypEG regression model (Casiraghi, 2017; Brandenberger et al. 2019). This is an inferential network model that allows for unbiased estimates of parameters when the independence assumption is violated (Casiraghi et al. 2016, 2017; Casiraghi, 2019). It is designed for multi-edge networks.

Our results indicate a weak association between online and offline support behavior. We show that party alliances mainly drive online endorsement. Whereas cosponsorship support is more rich in nature, showing significant effects of reciprocity dynamics and homophily traits,

\footnotetext{
${ }^{1}$ The term bill is used synonymously for any piece of legislation, including amendments, resolutions, interpellations, and parliamentary motions or postulates.
} 
online endorsement is more simplistic. This result calls into question the growing number of empirical studies that treat online data in the same manner as offline data.

This article is structured as follows: First, we discuss online and offline political support behavior, then we describe the two data sets analyzed, as well as the gHypEG regression model. Results are presented for four different models, technical details are given in the supplementary material. In our concluding remarks, we discuss the insights obtained from our analysis, and challenge the usefulness of online data to study real-world politics.

\section{Political Support Among Members of Parliament}

Members of parliament (MPs) know different ways of supporting or endorsing each other. They can lend votes to bills or proposed changes thereof, cosponsor newly introduced bills, covote, support each other in speeches, or host press events together (see, for instance Burstein, Bauldry and Froese, 2005, Wilson and Young, 1997; Talbert and Potoski, 2002, Peoples, 2008; Desmarais et al. 2015). By building alliances and exchanging support, MPs increase their visibility and their power to influence the decision-making process in their favor. The influenced decisions can then be communicated to their constituents, increasing their probability of reelection. As such, gathering and bestowing social support can be seen as a key mechanism guiding MPs' daily business.

Every collaboration or instance of support can increase trust between MPs (e.g., Friedkin, 2004: Melamed and Simpson, 2016), resulting in complex interdependencies among MPs. These interdependencies can be described as interwoven webs among MPs (Granovetter, 1985) or social networks of MPs with distinct community structures (Fowler, 2006; Kirkland and Gross 2014).

These instances of support among MPs are not limited to parliamentary actions. Outside parliament, MPs create bonds, for instance, through shared memberships in organizations, shared board memberships, or through personal interactions (Fischer et al., 2019, Desmarais et al. 2015). Moreover, with the increasing importance of online interactions on political debates and political vote-seeking, online political endorsement can help foster bonds between MPs that later result in parliamentary alliances.

While studies on online political behavior are abundant, little is known about the strategies MPs use to influence decision-making processes in the real world with online campaigns and interactions (Cook, 2016). An open question remains of how political behavior online differs 
from behavior offline. How do MPs interact and endorse each other online, and how much of this online behavior reflects the complex interdependencies built and groomed in the real world, i.e., offline? We address this question by comparing online and offline political support among Swiss members of parliament.

\section{Offline political support}

One of the most widely studied forms of legislative support is cosponsorship signatures (e.g., Campbell, 1982; Kessler and Krehbiel, 1996; Fowler, 2006, Kirkland, 2011; Bratton and Rouse, 2011). These low-cost, fast actions encode support among MPs for their proposed pieces of legislation. This support can either be directed at the content of a bill, which an MP finds worthy of support, or directed at the sponsor of a bill, with whom the cosponsor shares personal or strategic ties. Alternatively, during the preparation of a legislative bill, sponsors often approach other MPs to sway them to their cause, targeting MPs they trust or whose interest in the issue is known to the sponsor.

Whilst the number of cosponsorship signatures on a legislative proposal does not guarantee its success (for empirical evidence or lack thereof see Wilson and Young, 1997; Woon, 2008; Burstein, Bauldry and Froese, 2005, Krehbiel 1995), they are widely considered important signals among MPs (both for sponsors and cosponsors) (Mayhew, 1974).

This signaling can either be for the benefit of revealing your position to other MPs or to signal an MP's interest in an issue to their constituents. Kessler and Krehbiel (1996) addressed the question of whether legislative cosponsorship is driven by i) intralegislative signaling (Wilson and Young, 1997) or ii) extralegislative position taking (Schiller, 1995) and give empirical evidence that cosponsorship is driven by intralegislative signaling. Apart from intralegislative signaling, MPs issue cosponsorship support to build bridges to different parliamentary clusters (or groups) (Wilson and Young, 1997, Talbert and Potoski, 2002; Kirkland, 2011; Cranmer and Desmarais, 2011, Kirkland and Gross, 2014) or to reciprocate a favor (Brandenberger, 2018). Furthermore, multiple quantitative studies have shown that homophily plays an important role, indicating that MPs cosponsor bills of similar MPs more readily than MPs with whom they do not share traits (Craig et al. 2015; Bratton and Rouse, 2011; Tam Cho and Fowler, 2010). Party, race, gender, and state homophily are amongst the most important correlates with cosponsorship activities. These motivations to cosponsor a bill are by no means universal nor do they apply to all members equally. For instance, Brandenberger (2018) shows that 
for the 113th U.S. House of Representatives, favor trading is more common among Republican members of Congress.

Thus, legislative support has been shown to encompass a broad variety of motivations, ranging from individual ideological preferences to signaling and group dynamics. The question remains whether online political endorsement among the political elite i) is motivated by internal or external factors and ii) likewise guided by a wide variety of individual and network effects.

\section{Online political endorsement on social media platforms}

Political actors have expanded the political arena to online platforms, where they debate current events, comment on campaigns, or present themselves and their political positions. This raises the question of whether the online behavior of political elites differs from behavior offline. The question is relevant because online data is more easily accessible and is currently widely used to study the behavior of politicians and political actors. However, this also poses a problem because the external validity of political actors' online behavior data may not be a given. It raises the question of whether online endorsement among MPs reflects their willingness to cooperate in offline political arenas. Few studies directly link online and offline political behavior to study potential correlations among the two.

Cook (2016) studies three forms of Twitter communications and compares explanatory factors to factors driving congressional co-voting and cosponsorship in the U.S. Senate and the Main State Legislature. For each pair of MPs, he examines whether direct mentions between the two MPs, their number of shared mentions, or the number of co-mentions by others are guided by party homophily, geographic, and other forms of homophily and shared committee service. He finds that party homophily is weaker in online social behavior than in legislative behavior, both for federal and state legislators. Regrettably, Cook 2016) does not match online and offline behavior in a statistical model to examine the interplay between the two behaviors. Furthermore, even though Cook (2016) uses a network inference model (QAP regression), he does not control for higher order effects on pairs (i.e., triadic closure), which potentially bias his results (Cranmer and Desmarais, 2011).

Cherepnalkoski and Mozetič (2016) examine the re-tweeting behavior of members of the European Parliament over one year. They use a cluster analysis (Louvain algorithm) to divide the Twitter network into communities and find that offline political groups in the European 
Parliament are mirrored in these online communities. They assess this matching between offline political groups and re-tweet communities using precision and recall measures for each European MP. Cherepnalkoski et al. (2016) extended the previous study to examine whether re-tweeting behavior matches co-voting patterns among the same set of European MPs. Specifically, they use Exponential Random Graph modeling (ERGM) to examine whether re-tweeting is correlated with roll-call voting. Since ERGMs were developed for binary networks (i.e., networks where the links or edges between nodes are either present $(=1)$ or absent $(=0))$, they disaggregate each vote $(N=2,535)$ into separate co-voting networks and study the correlation between each co-voting incident and online endorsement via re-tweeting activities on Twitter. While their ERGMs report positive, significant effects for the correlation between retweeting and co-voting, their effect sizes are near zero, indicating only a weak correlation between the two 2

The results of these limited studies raise the question of whether politicians' online behavior systematically differs from offline behavior. While the studies show that some positive correlation between online and offline behavior can be expected, important open questions remain: Can online political endorsement be seen as a form of extra-legislative signaling, bringing an additional dimension to explaining offline support? Is online political endorsement as complex as offline support, i.e., does it reflect the interdependencies among MPs to form triads, reciprocate favors, or group together based on similar attributes? This paper tackles these open questions. We are particularly interested in dissecting the different factors that drive offline political support behavior among MPs and examining whether online political endorsement differs from offline support.

\section{Data and Methods}

\section{Data}

Our study uses two different data sets to compare online and offline support among members (MPs) of the Swiss National Council, the lower chamber of the Swiss parliament.

Our offline data set contains information about cosponsorship among MPs for two consecutive legislative periods from December 2007 to 2015. During this period 11 different parties

\footnotetext{
${ }^{2}$ Cherepnalkoski et al. (2016) do not report the control variables they used in their ERGMs in detail, making it difficult to assess whether their models are well-specified and therefore unbiased due to omitted variables.
} 
(see Supplementary Information for a list of members and their party affiliation) were represented in parliament. ${ }^{3}$ From December 2007 to 2015, 321 MP served in the National Council (200 per session, with some overlap between sessions).

In total, there were 91, 225 cosponsorship events between the $321 \mathrm{MPs}$, with the average MP cosponsoring 284.2 bills (median $=174$, sd $=323.3$ ) over the two legislative periods. The effort involved in cosponsoring a bill in parliament can range from co-writing the bill (i.e., using significant time and resources in the bill drafting stage) to signing a bill on the way to the next parliamentary session (i.e., spending 5 seconds on a signature).

Our online data set contains information about endorsement clicks among users of Politnetz. This online platform for political debate and networking was active from 2009 to 2016, to foster the political exchange bewteen regular citizens and politicians in Switzerland. On the platform, users (politicians and citizens) could (i) publish posts on political issues and 'comment' on posts of other users; (ii) 'like' the posts of others, and (iii) publicly declare support for a politician by pressing an 'endorse'-button on the politician's profile page (Garcia et al. 2015).

The effort for an 'endorse'-click on social media is comparable to the effort for a cosponsorship signature. It can range from maintaining a strong social media relationship to identifying an ally in short time and clicking the 'endorse'-button. The platform does not suggest to support certain politicians to the users based on their previous support actions, making 'endorse' clicks on Politnetz free from algorithmic manipulations (as opposed to Twitter, for example).

Overall, 3, 030 users were active on Politnetz between 2009 and 2016 and shared 21,347 endorsements. After matching these data with the cosponsorship data set, we identified 163 MPs from the Swiss parliament who were active on Politnetz between 2009 and 2016. We focus our analysis on these 163 MPs. In the Swiss parliament, these 163 MPs cosponsored each other 26, 559 times. On Politnetz, the same 163 MPs shared 55 direct support links.

In order not to bias our results by subsampling our observations, we take two countermeasures: (1) We calculate the online endorsement similarity among the 163 MPs based on the endorsements expressed towards any of the 3,030 users of Politnetz, not only among themselves. This gives us a more robust measure of online endorsement behavior among MPs. (2) We calculate the in- and outgoing shared partner statistics of the 163 MPs accounting for support signatures issued to or from any of the 321 total members of parliament. Thus, considering

\footnotetext{
${ }^{3}$ It should be noted that the Free Democratic Party (FDP) merged with The Liberals at the beginning of 2009. We treat them as one party (i.e., as FDP.The Liberals as they are known from 2009 onwards) during our observation period.
} 
Why Online does not Equal Offline: Comparing Online and Real-World Political Support Among Politicians.

the two legislative periods, we do not reduce this endogenous statistic to only cosponsorship signatures among the 163 MPs. We detail these two countermeasures below when describing the operationalization of covariates.

\section{Method}

Political support among MPs in the form of cosponsorship is an inherently social act. One MP $i$ supports another MP $j$ by signing bills sponsored by the latter. We operationalize this as a directed multi-edge graph $\mathcal{G}=(V, E)$ with $|V|=163$ vertices and $|E|=26,559$ edges. The vertices, or nodes, represent the MPs, the edges their cosponsorship signatures. The matrix $A$ denotes the adjacency matrix of $\mathcal{G}$, whose entries $A_{i j} \in \mathbb{N}_{0}$ correspond to the number of times MP $i$ signed a bill sponsored by MP $j$. Because cosponsorship is directed from $i$ to $j$, the adjacency matrix $A$ defines a directed graph, with $A_{i j}$ possibly different from $A_{j i}$.

Such a network representation is necessary to reflect the fact that cosponsorship signatures depend on another Fowler (2006); Cranmer and Desmarais (2011). Specifically, the cosponsorship behavior of one MP may depend on partisanship, or whether members have signed or not signed particular bills before Bratton and Rouse (2011); Craig et al. (2015). Similarly, an MP may tend to reciprocate the cosponsorships support received Brandenberger (2018). For this reason, this dataset cannot be studied as a collection of independent observations.

We are interested in how offline cosponsorship support can be explained by online endorsement among MPs. Statistical regression models allow to perform such analysis, by regressing offline cosponsorship support (our dependent variable) against online endorsement. Conventional regression models on network data, though, are unreliable. The intrinsic dependencies between observations in our data violate the fundamental i.i.d. assumption of these models. This leads to erroneous estimates of the model's standard errors Cranmer and Desmarais. 2011).

To avoid this known problem, in this article we use an inferential network model. Inferential network models first define a baseline random graph model that generates edges between all different nodes uniformly. Then, they incorporate covariates with different weights to increase the probability of creating some edges instead of others. Finally, fitting these weights, i.e., the model parameters, to the observed data allows to estimate the explanatory power carried by these covariates. 
Following this approach, we define a baseline model for offline cosponsorship support. Under this baseline, cosponsorship signatures between any pair of MPs are assumed to be equiprobable. The maximum number of cosponsorship signatures a MP $i$ can give to a MP $j$ is the number of bills $n_{j}$ that MP $j$ has sponsored. This fact constrains the maximum number of possible edges that can exist between any given pair of MPs. Thus, we can see such a baseline network model as the uniform sampling of $|E|$ signatures from the possible cosponsorships $\Xi=\left\{\Xi_{i, j}=n_{j}\right\}_{i, j \in V}$ between all MP pairs $i, j$. The matrix $\Xi$ allows to represent the possible signatures for all pairs in a compact form.

Such a simplistic baseline model, however, is insufficient to describe the observed data because real cosponsorship support is not uniformly distributed. Multiple factors are known to shape the cosponsorship behavior of MPs, like party membership or homophily and social bonds between sponsor and cosponsors. These explanatory factors can be introduced in the form of sampling weights for different edges that bias the uniform edge generating process.

The main explanatory factor we are interested in is online endorsement (OE) and its weight $\theta_{O E}$ on cosponsorship. It reflects how much the probability of observing a signature between MPs $i$ and $j$ is biased by the fact that $i$ has endorsed $j$ online. To quantify the weight $\theta_{O E}$ of such a categorical explanatory factor $h_{i j}^{(O E)}$, or covariate, we employ the generalized hypergeometric ensemble of random graphs (gHypEG, see Casiraghi and Nanumyan 2018 for a more formal presentation).

Similar to the matrix $\Xi$, we write the covariate associated with online endorsement in matrix form: $\boldsymbol{h}^{(O E)}=\left\{h_{i, j}^{(O E)}\right\}_{i, j \in V}$. This is the independent variable of our inferential network model. According to the gHypEG, the probability of observing the graph $\mathcal{G}$ with adjacency matrix $\boldsymbol{A}$ depends on both the matrix $\Xi$ and the matrix $h^{(O E)}$ as follows:

$$
P\left(\boldsymbol{A} \mid \boldsymbol{\Xi}, \theta_{O E}, \boldsymbol{h}^{(\boldsymbol{O E})}\right)=\prod_{i j}\left(\begin{array}{c}
\Xi_{i j} \\
A_{i j}
\end{array}\right) \int_{0}^{1} \prod_{i j}\left(1-\exp \left\{\frac{\left(h_{i j}^{(O E)}\right)^{\theta_{O E}}}{S} \log z\right\}\right)^{A_{i j}} d z
$$

where $S=\sum_{i j} h_{i j}^{(O E)^{\theta_{O E}}}\left(\Xi_{i j}-A_{i j}\right)$. The weight $\theta_{O E}$ of the covariate $h^{(O E)}$ is estimated by means of maximum likelihood (Casiraghi, 2017). Thanks to gHypEG, we are now able to model repeated interactions such as cosponsorship signatures. This is in stark contrast with other inferential network models that require reducing the interaction counts to a binary variable, usually by introducing a cut-off threshold. 
In the model given by Eq. 11), $\theta_{O E}$ represents the log-odds of observing a cosponsorship signatures between any directed pair of MPs for which an online endorsement has been observed, against a directed pair for which no endorsement has been observed Thus, estimating $\theta_{O E}$ allows to quantify how much offline cosponsorship support is explained by online endorsement.

To get a reliable estimate of $\theta_{O E}$, though, we need to account also for known effects that drive offline cosponsorship support. These could mask the main effect we are interested in (Spector and Brannick, 2011). To include these known effects into the model, in the next section we specify endogenous $\left\{h_{i j}^{\left(e_{l}\right)}\right\}_{l}$ and exogenous $\left\{h_{i j}^{\left(o_{l}\right)}\right\}_{l}$ control variables. Ultimately, this means that the relative sampling weight $\omega_{i j}$ of a cosponsorship signature between $i$ and $j$ depends not only on the online endorsement $h_{i j}^{(O E)}$, but also on these additional effects. The odds of observing a signature between a pair $i, j$ of MPs instead of a second pair $k, l$ are therefore given by $\omega_{i j} / \omega_{k l}$. In the simple case described above, we have:

$$
\log \omega_{i j}=\theta_{O E} \log h_{i j}^{(O E)}
$$

i.e., the log-odds of observing a cosponsorship signature are proportional only to the covariate $h_{i j}^{(O E)}$ representing online endorsement. In the general case, we extend the log-odds such that they are proportional to all covariates:

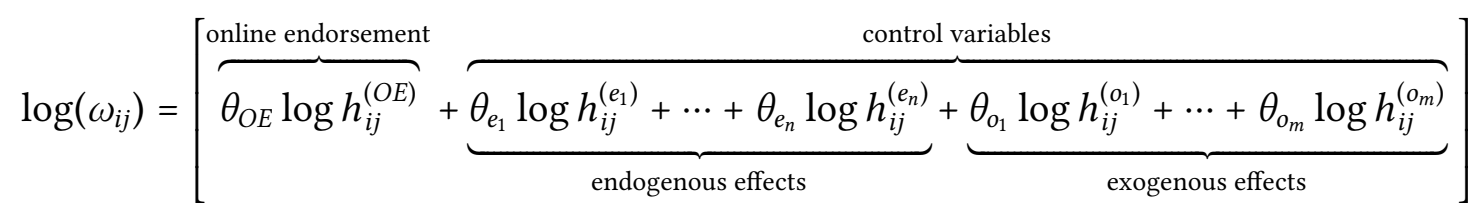

Eventually, we replace $\left(h_{i j}^{(O E)}\right)^{\theta_{O E}}$ in Eq. 11 with $\omega_{i j}$, to obtain the full inferential network model (Casiraghi, 2017).

Similar to a regular statistical regression model, we estimate simultaneously all parameters $\theta_{x}$ for each of the covariates $\boldsymbol{h}^{(\boldsymbol{x})}$. This is achieved using a numerical Maximum Likelihood Estimation (MLE) (Casiraghi 2017). Specifically, we use the ghypernet package Casiraghi and Nanumyan (2020) in the statistical environment R (R Core Team 2020) to estimate the gHypEG inferential model.

\footnotetext{
${ }^{4}$ Assuming the two pairs have identical $\Xi$ values.
} 


\section{Operationalization of Covariates}

\section{Independent Variable: Online endorsement}

We operationalize direct online endorsement in the form of a matrix $\boldsymbol{h}^{(O E)}$ representing a categorical variable. Entries $h_{i j}^{(O E)}$ are either 1 when no online endorsement between two MPs has been recorded or $e$ if MP $i$ supported MP $j$ online. When we plug this operationalization in Eq. (3), the log-odds of observing a signature between a pair $i, j$ with online endorsement against a pair $k, l$ without online endorsement is exactly $\theta_{O E}$ as required:

$$
\log \frac{\omega_{i j}}{\omega_{k l}}=\log \omega_{i j}-\log \omega_{k l}=\theta_{O E} \log h_{i j}^{(O E)}-\theta_{O E} \log h_{k l}^{(O E)}=\theta_{O E}
$$

Eventually, we have to avoid biases resulting from our sample's restriction to 163 MPs. Therefore, we also generate a similarity matrix for the online endorsement behavior. This similarity matrix measures the overlap between the lists of supportees for both MPs $i$ and $j$. For each pair $i, j$ we measure the percentage of $i$ 's supportees $S_{i}$ that is shared by $j$ :

$$
h_{i j}^{(O E \operatorname{sim})}=\frac{\left|S_{i} \cap S_{j}\right|}{\left|S_{i}\right|}
$$

$S_{i}$ and $S_{j}$ refer to the set of politicians that MPs $i$ and $j$ endorse online (i.e., $S_{i}=i$ 's supportees). $h_{i j}^{(O E s i m)}$ results in a directed similarity measure between MPs $i$ and $j$, that scales from 0 , most dissimilar, to 1 , most similar. A value of 1 indicates that both MPs $i$ and $j$ endorse the exact same politicians online. For example, if $i$ supports five politicians $(a, b, c, d$, and $e$ ) and $j$ supports 3 politicians $(a, f, g)$, then $h_{i j}^{(O E s i m)}=0.2$ and $h_{j i}^{(O E s i m)}=0.33$. By doing so, we ensure that $h_{i j}^{(O E s i m)}$ accounts for the number of endorsement clicks an MP gives $5^{5}$ We now use $h^{(\text {OEsim) }}$ to replace the independent variable $\boldsymbol{h}^{(\mathrm{OE})}$ in Eq. 3 .

\section{Control Variables}

We control for several endogenous and exogenous effects. Cosponsorship dynamics have been shown to be guided by endogenous effects, such as reciprocity dynamics (or favor trading),

\footnotetext{
${ }^{5}$ As a sensitivity check, we also operationalized similar online behavior using the Jaccard similarity. This measure is not affected by the number of politicians an MP supports on Politnetz. As results did not vary between the two measures, we perform our primary analysis with the simpler similarity measure presented above. We report our operationalization of the Jaccard similarity, as well as the results in the SI Online.
} 
closure, and homophily effects. We calculate the relevant endogenous network effects on the full cosponsorship network, i.e., with $321 \mathrm{MPs}$ and 91, 225 cosponsorship signatures. As for exogenous effects, we use an MP's party membership, the canton they represent as well as their gender to check for homophily traits between pairs of MPs.

For each pair $i, j$, of MPs we measure whether sponsors $j$ have a tendency to reciprocate support they received form $i$ :

$$
h_{i j}^{\text {(recip) }}=A_{j i}
$$

where $A_{j i}$ refers to the number of signatures between $j$ and $i$.

We also control for the fact that sharing multiple supporters builds trust among these MPs, which makes them more likely to support each other's bills. This effect is known as social closure (Simmel, 1908 (1950, Coleman, 1990, Burt and Knez, 1995, Granovetter, 1973) and can be operationalized via triadic closure among MPs. We measure two different forms of triadic closure: The first one measures whether MP $i$ is more likely to support MP $j$ because they both supported MP $k$. If $i$ and $j$ continuously support the same MPs, this may signal $i$ and $j$ that their interests and positions are aligned. This behavior, in turn, increases the probability of $i, j$ directly supporting each other. We call this effect outgoing shared partners:

$$
h_{i j}^{\text {(out-sp) }}=\sum_{k} \min \left(A_{i k}, A_{j k}\right)
$$

where $k$ refers to each of shared partners MP $i$ and $j$ have. Since there are multiple edges between pairs, we take the minimum between $A_{i k}$ and $A_{j k}$ to ensure that only closed triads are counted (see Brandenberger et al. 2019).

The second measure of triadic closure accounts for the event that if MP $i$ and MP $j$ are both supported by MP $k$, they also support each other. We call this effect incoming shared partners:

$$
h_{i j}^{(\mathrm{in}-\mathrm{sp})}=\sum_{k} \min \left(A_{k i}, A_{k j}\right)
$$

where $k$ refers to the number of shared partners MP $i$ and $j$ have. Since there are multiple edges between nodes, we take the smallest number between the incoming pairs $k, i$ and $k, j$.

We further account for different homophily effects, using two different operationalizations, depending on whether the homophily variable is categorical or continuous. For the continuous 
case, we include an absolute difference term:

$$
h_{i j}^{(\text {abs-diff) }}=\left|x_{i}-x_{j}\right|,
$$

where $x$ refers to the continuous scaled variable in question. We control for age difference using this absolute difference effect. For the categorical case, we include a matching homophily term:

$$
h_{i j}^{\text {(homophily) }}:= \begin{cases}e & \text { if } x_{i}==x_{j} \\ 1 & \text { otherwise }\end{cases}
$$

Using this term, we further test for party, cantonal (or state), and gender homophily as well as shared committee membership to account for increased cosponsorship activity between two MPs (see Casiraghi, 2019). Again, such an operationalization allows to interpret the respective model parameter of each categorical variable as a log-odds (cf. Eq. (4)).

To ensure the results are not biased, we further include a difference measure for the difference in the number of supportees between $i$ and $j$ as a control variable. We operationalize this as

$$
h_{i j}^{(\text {OEdiff })}=\left|S_{i}-S_{j}\right|
$$

Finally, we introduce correcting factors that provide a baseline for those pairs for which a given covariate provides no information. For example, in the case of the independent variable $\boldsymbol{h}^{\text {(OEsim) }}$, we introduce an extra control variable $\boldsymbol{b}^{\text {(OEsim) }}$ which corrects the log-odds estimation of $\boldsymbol{h}^{\text {(OEsim) }}$ by introducing a baseline probability for those pairs $k, l$ that share no online endorsees, and for which it is then impossible to compute a similarity value. For any covariate $\boldsymbol{h}^{(\boldsymbol{x})}$ needing correction, we thus construct such an extra control variable $\boldsymbol{b}^{(\boldsymbol{x})}$ in the following way:

$$
b_{i j}^{(x)}:= \begin{cases}e & \text { if } h_{i j}^{(x)}=\varnothing \\ 1 & \text { otherwise. }\end{cases}
$$

Furthermore, we modify the original covariate $\boldsymbol{h}^{(\boldsymbol{x})}$ to allow for the baseline correction:

$$
h_{i j}^{(x)}:= \begin{cases}1 & \text { if } h_{i j}^{(x)}=\varnothing \\ h_{i j}^{(x)} & \text { otherwise. }\end{cases}
$$


These two definitions allows us to effectively introduce a baseline sampling weight for all pairs $k, l$ for which no data is available for the given covariate $h^{(\boldsymbol{x})}$ (Casiraghi, 2017):

$$
\log \omega_{k l}=\theta_{x} \log h_{k l}^{(x)}+\phi_{x} \log b_{k l}^{(x)}=0+\phi_{x}
$$

where $\theta_{x}$ and $\phi_{x}$ are the respective model parameters for $\boldsymbol{h}^{(\boldsymbol{x})}$ and $\boldsymbol{b}^{(\boldsymbol{x})}$.

\section{Results and Discussion}

Table 1 reports the results of the gHypEG regression on cosponsorship support. We report four different models. Model (1) checks for the correlation between direct online and offline support. Model (2) checks for the correlation between offline support and online endorsement similarity. Model (3) reports the correlation between online and offline support under the control of different variables that have been shown to influence cosponsorship signatures. Model (4) reports the correlation between online and offline support while controlling only for partisanship and reciprocity.

In Model (1), results indicate that there is a positive correlation between online and offline political support. MPs who directly endorse each other online on Politnetz also cultivate a supportive relationship offline. The odds of MP $i$ cosponsoring the bill of MP $j$ increase by a factor of $1.9\left(e^{0.621}=1.86\right)$ if $i$ endorses $j$ online. Interestingly, online endorsement cannot account for the variance in cosponsorship activities (see near-zero pseudo- $R^{2}$ for Model (1)). This result indicates that even though there is a positive relationship between online and offline support, offline support presents itself as more complex than online endorsement. Since our sample consists of MPs active in Parliament and on Politnetz, the results of direct online endorsement may be distorted.

To alleviate this bias, we operationalize online endorsement as endorsement similarity and test its correlation with offline support. By including the similarity measure of online endorsement, we see that the positive (and significant) effect holds. Even more importantly, around $22.7 \%$ of the variation of offline cosponsorship support is explained through online behavior. Increasing the similarity from 0 (no similarity among online endorsementees between two MPs) to 0.5 (moderate similarity, $50 \%$ of supportees in common), increases the odds of cosponsorship support from $i$ to $j$ by a factor of $7\left(0.5^{0.771} / e^{-2.492}=7.08\right)$. This seven-times increase in the odds, if online endorsementee similarity increases from dissimilar to moderately simi- 
Table 1: gHypEG regression on cosponsorship signatures

\begin{tabular}{|c|c|c|c|c|}
\hline & (1) & $(2)$ & (3) & $(4)$ \\
\hline \multicolumn{5}{|l|}{ Online behavior } \\
\hline Direct online endorsement & $0.621(0.078)^{* * *}$ & & & \\
\hline Online endorsement similarity & & $0.771(0.007)^{* * *}$ & $0.041(0.009)^{* * *}$ & $0.068(0.009)^{* * *}$ \\
\hline \multicolumn{5}{|l|}{ Endogenous variables } \\
\hline Reciprocity (weighted) & & & $0.267(0.008)^{* * *}$ & $0.378(0.007)^{* * *}$ \\
\hline Shared partners (incoming, weighted) & & & $0.097(0.009)^{* * *}$ & \\
\hline Shared partners (outgoing, weighted) & & & $0.038(0.008)^{* * *}$ & \\
\hline Shared committees (weighted) & & & $0.071(0.027)^{* *}$ & \\
\hline \multicolumn{5}{|l|}{ Homophily } \\
\hline Gender homophily & & & $-0.020(0.013)$ & \\
\hline Age difference & & & $-0.096(0.007)^{* * *}$ & \\
\hline Party homophily & & & $1.154(0.020)^{* * *}$ & $1.027(0.020)^{* * *}$ \\
\hline State (=Canton) homophily & & & $0.416(0.020)^{* * *}$ & \\
\hline \multicolumn{5}{|l|}{ Control } \\
\hline Online endorsement activity & & $-0.041(0.005)^{* * *}$ & $-0.030(0.006)^{* * *}$ & $-0.015(0.006)$ \\
\hline \multicolumn{5}{|l|}{ Correcting factors (dummies) } \\
\hline Online endorsement similarity (dummy, vs. no support) & & $-2.492(0.020)^{* * *}$ & $-0.099(0.027)$ & $-0.201(0.028)^{* * *}$ \\
\hline Online endorsement activity (dummy, vs. no activity) & & $0.007(0.053)$ & $-0.004(0.053)$ & $-0.005(0.053)$ \\
\hline Reciprocity (dummy, vs. no reciprocity) & & & $-0.952(0.020)^{* * *}$ & $-1.007(0.020)^{* * *}$ \\
\hline Shared partners (incoming, dummy vs. no partners) & & & $-0.013(0.018)$ & \\
\hline Shared partners (outgoing, dummy vs. no partners) & & & $-0.171(0.017)^{\star * *}$ & \\
\hline Shared committees (dummy, vs. no committees) & & & $-0.119(0.014)^{* * *}$ & \\
\hline Age difference (dummy, vs. no difference) & & & $0.036(0.036)$ & \\
\hline AIC (relative) & 40523.916 & 20342.138 & 0.000 & 1295.242 \\
\hline McFadden pseudo $-R^{2}$ & 0.029 & 0.227 & 0.428 & 0.415 \\
\hline
\end{tabular}

lar, also coincides with some explanatory power over offline support. However, the explained variation is not as large as it could be, indicating that offline political support dynamics are richer and more complex than online political endorsement dynamics.

Our third model includes a range of control variables previously used to explain cosponsorship dynamics. Upon the inclusion of these additional explanatory variables, the online endorsement similarity effect reduces to near zero, indicating a strong correlation among online endorsement similarity and one (or multiple) control variables. The odds of MP $i$ cosponsoring MP $j$ if their online similarity increases from negligible to moderate only increase by a factor of $1.07\left(0.5^{0.041} / e^{-0.099}=1.07\right)$.

Figure 1 reports marginal effects of online endorsement similarity on cosponsorship tie probabilities. Figure 1(a) is based on Model (2) and depicts the positive effect of online endorsement on offline support. The support is non-linear, with a steeper increase as online similarity increases from $0 \%$ to $25 \%$ endorsement similarity. Figure 1(b) is based on Model (3) and shows 
(a) Model (2)

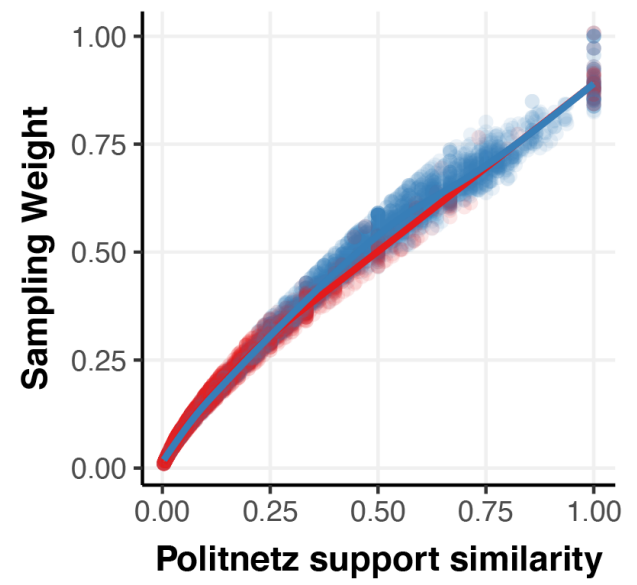

(b) Model (3)

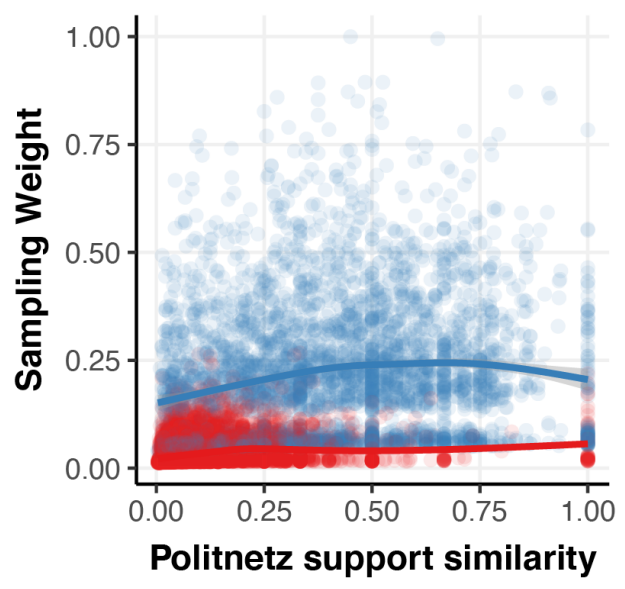

Within party

Figure 1: Marginal effects of endorsing each other online on probability of cosponsoring each other's bills offline. (a) is based on model (2) in Table 1 (b) is based on Model (3) in Table 1 The effect of online endorsement similarity is reduced to near-zero upon the inclusion of control variables for offline cosponsorship. Party homophily mainly drives the positive effect of online similarity.

how the online endorsement similarity effect reduces to a near-zero effect upon the inclusion of additional parameters. We tested which of the control variable masks the online endorsement similarity. Model (4) shows that high predictive values of online political endorsement on offline support are mainly driven by party homophily (i.e., a form of partisanship).

Model (4) shows that by controlling offline support with party homophily and reciprocity, the online endorsement effect diminishes to a near-zero effect. The estimates of Model (4) indicate the strong correlation between party homophily and online endorsement, indicating that online endorsement is mainly driven by party homophily. This result is surprising for two reasons: (i) Switzerland is a consensus democracy with a weak party structure. Previous studies indicate that adhering to party lines and party unity is less crucial in the Swiss political landscape (Schwarz, 2009, Bailer and Bütikofer, 2015). The fact that it presents as strongly in online political behavior is contradictory and warrants additional analyses. (ii) The substantial reduction in effect size for online endorsement by the inclusion of only party homophily shows that online endorsement dynamics are less varied and not as complex as offline support.

Figure 2 shows two heat plots for online and offline support similarities. Parties are ranked 
(a) Avg. Online Party Support Similarities

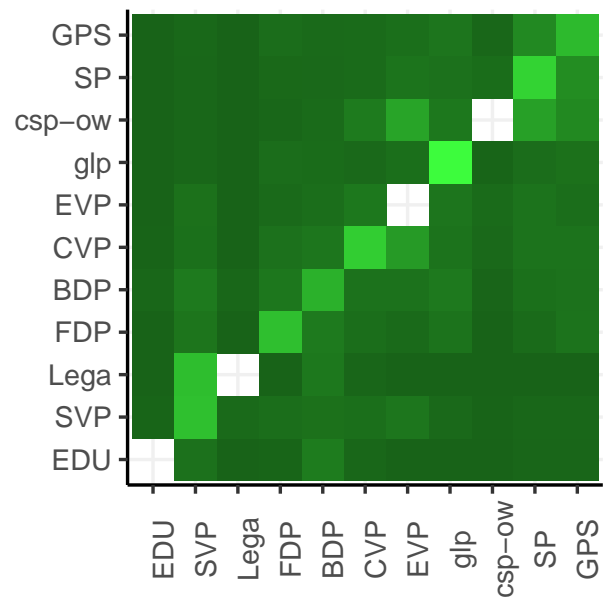

Avg. online support similarity

$\begin{array}{lllll}0.1 & 0.2 & 0.3 & 0.4 & 0.5\end{array}$ (b) Avg. Shared-\% Party Cosponsorships

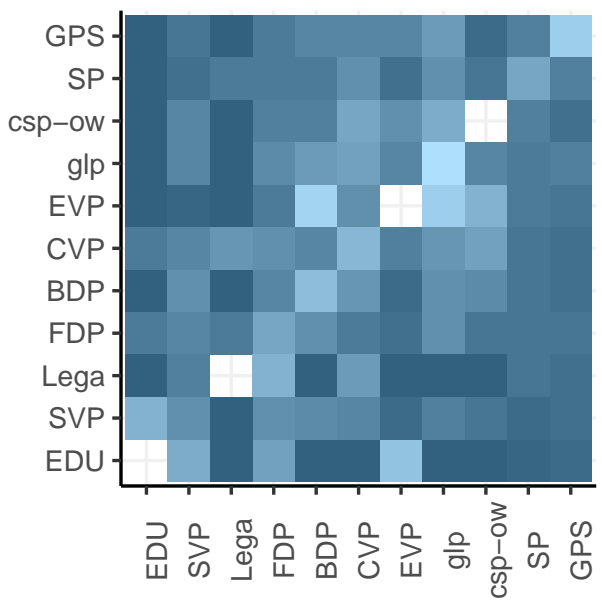

Avg. co-sponsorship support (\%-share)

$\begin{array}{lllll}0.00 & 0.05 & 0.10 & 0.15 & 0.20\end{array}$

Figure 2: Heatplot for within and between party support. (a) represents average online endorsement similarity between MPs and (b) represents average share of cosponsorship support among MPs within and between different parties. csp-ow, EVP, Lega and EDU only had one representative in parliament and no similarity score is calculated for themselves.

from most right (EDU, SVP) to most left parties (SP, GPS). Figure 2(a) shows average online similarities between parties. The higher values are obtained for the diagonal for online similarity, indicating strong partisanship in online endorsements. Figure 2(b) shows the average share of cosponsorship signatures between parties. Here, the diagonal is less prominent, indicating that within-party support plays less of a role in cosponsorship activities. This holds especially true for middle to right parties.

Beyond party homophily, there are still a few MPs who support the same politicians online, but are part of two different parties. Granted, these cases are rare (and therefore difficult to detect statistically), yet they exist. One example poses council members Louis Schelbert and Yvonne Feri. Both were active members of parliament between 2011 and 2015. Louis Schelbert is part of the green party (GPS), and Yvonne Feri is part of the left party (SP), representing both left parties in the Swiss parliament. They represent different cantons (Luzern and Aargau, respectively) but served on the same committee between 2011 and 2015 (Control Committee), explaining their online endorsement similarities. A second example poses Hansjoerg Hassler 
(right party, SVP) and Rosmarie Quadranti (right-to-middle party, BDP). They are part of the same parliamentary group (or fraction) since the parties share conservative leanings. During the time Rosmarie Quadranti served (2011-2015), Hansjoerg Hassler was president of the parliamentary group, possibly explaining their online endorsement similarities.

These two examples illustrate that some traces of parliamentary support are reflected in online endorsement behavior. Figures 3 and 4 take a closer look at online political endorsement similarities and their extreme values. Figure 3 shows which variables are indicative of high online endorsement similarity apart from party homophily. The top two plots are for MPs from different parties only (labeled between parties), the bottom two plots are for MPs who share the same party. Three homophily variables are tested to see if they facilitate inter-party similarities: We examine cantonal homophily, gender, and shared committee activities. Both within and between party pairs show slightly higher online similarity if both MPs come from the same canton (light blue boxplots). For pairs with high online similarity only (above 50\% match in supportees among two MPs), MPs from the same canton score an average similarity of 0.7, whereas MPs from different cantons average at around 0.65 (Welch two sample t-test, $t=-3.18, p-$ value $=0.0017)$. This difference holds for within and between party pairs, indicating that beyond party homophily, cantonal homophily plays a minor role in MP's choice of politicians to endorse online.

As for gender homophily, there is another small, but interesting pattern in the data. For pairs with high online similarity only, MPs from the same party show slightly more online endorsement similarity if both MPs claim the same gender. For pairs from different parties, the opposite is true. Here, the median online endorsement similarity for MPs from different parties and same gender is 0.55 , compared to MPs with different gender 0.67 (even though average similarities are almost the same, at 0.69 for differently gendered and 0.68 for same-gendered MP pairs, t-test, $t=0.17191, p$-value $=0.8638$ ). Another small driver of non-partisan support is sharing the same committee activity. Here, MPs with high online endorsement similarity have a higher average similarity if they shared a committee seat with the respective other MP (mean similarity of 0.66 for MPs who did not share committee membership versus mean similarity of 0.74 for MPs who have one or more committee assignments in common; t-test, $t=-2.1714, p-$ value $=0.033$ ). This difference is only visible for MPs with high online similarity.

Figure 4 examines whether age differences explain online endorsement similarities among MPs. Figure 4(a) indicates no correlation among age differences and online endorsement simi- 
(a) All pairs $(\mathrm{N}=26,406)$

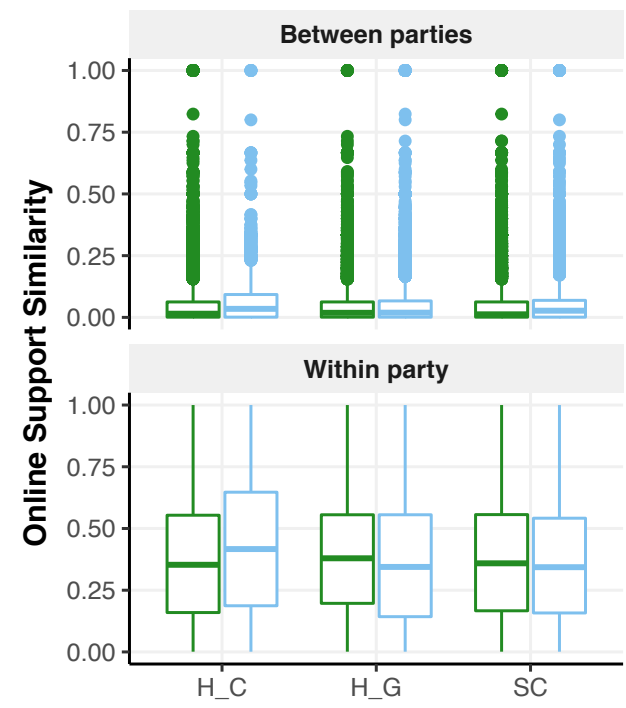

(b) Pairs with high online similarity $(\mathrm{N}=1,665)$

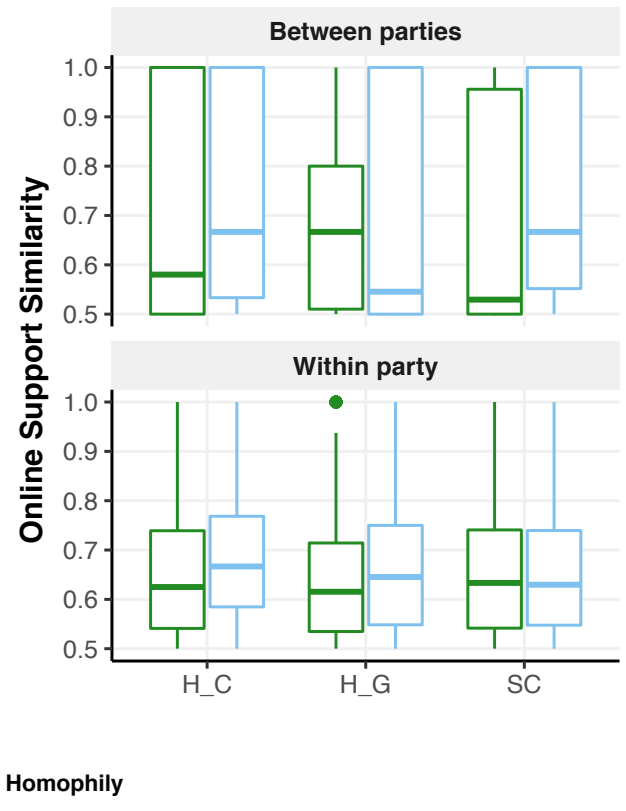

Figure 3: Boxplot showing association between online similarity and three different homophily variables. Left panels show all dyads (pairs of MPs), sorted for MPs who belong to different parties (top left), and MPs who belong to the same party (bottom left). Right panels show dyads with high online similarities (> 0.5). MPs of different parties with high online similarities also tend to belong to the same canton or sit on the same committees.

larity. In cases of high online endorsement similarity, shown in Figure 4 (b), it can be seen that MPs from different parties who are of a similar age (no more than an eight year age difference) show a significantly higher online endorsement similarity. Once the age difference increases from 10 to 30 years, both MPs-pairs from the same or different parties have similar online endorsement values.

It has to be noted that all these effects are small in size and are only used here to examine potential other drivers of online endorsement similarity. Compared to party homophily, none of these other drivers comes close in size and power, indicating that Swiss MPs present strong party unity online. However, some traces of offline support are mirrored in online endorsement. 
(a) All pairs $(\mathrm{N}=26,406)$

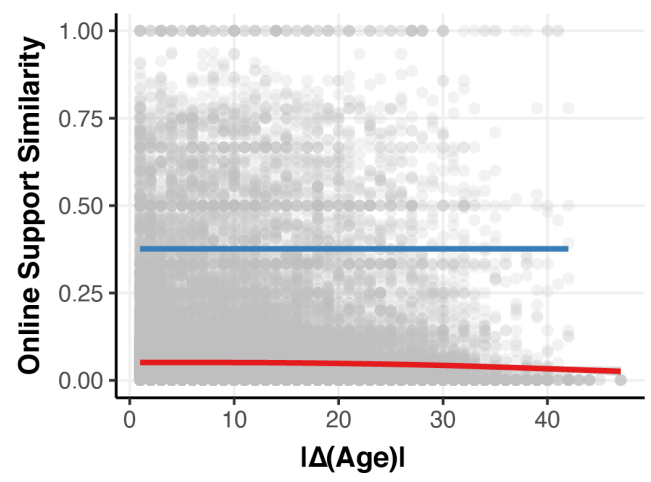

- Between parties (b) Pairs with high online similarity $(\mathrm{N}=1,665)$

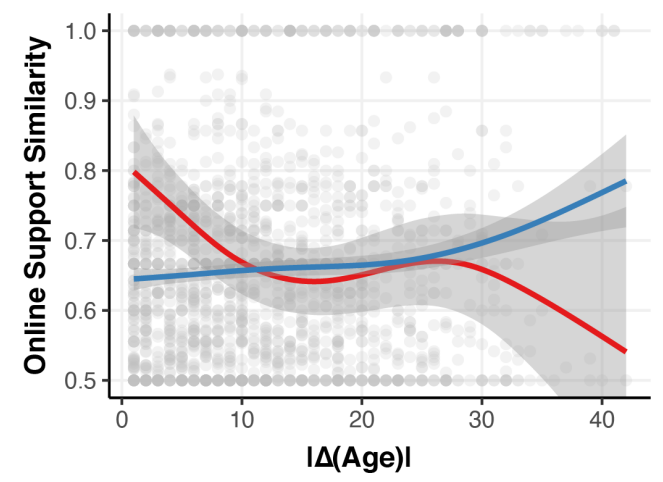

Within party

Figure 4: Scatterplot showing association between online similarity and age differences between MPs. Left panel shows all dyads, right panel only dyads with high online similarities (> 0.5$)$. MPs of different parties seem to have slightly higher online similarities the closer they are in age. However, the association only holds for dyads with high online similarities and is not robust.

\section{Conclusion}

The external validity of data on online political behavior is not always given. We examined online and offline political support among MPs and found little evidence that data on online political behavior is informative of offline behavior. Notably, online political endorsement amongst MPs cannot explain cosponsorship support amongst MPs and is mainly driven by party homophily. Our results indicate that online political endorsement is less varied or complex and reflects only a specific aspect of offline behavior.

These results question the extent to which online political behavior can be used as a proxy for offline or real-world political behavior. Do politicians present themselves differently online? Can analyses performed with online data on political behavior, for instance, to examine coalition formation or political ideology, be trusted to represent a politician's behavior in the real world?

There are several limitations to the present study that call for additional research on the external validity of online data on political behavior. First, our analysis is cross-sectional, overlapping two data sets from around the same time, 2007-2015 for the parliamentary cosponsorship data and 2009-2016 for the online Politnetz data. Both data sets are temporal: cosponsorship signatures are submitted on specific dates and time-stamps of online endorsement instances 
are available. Even though, it is impossible to causally link the two data sets, which is why we resorted to a cross-sectional analysis. Weaver et al. (2018 p. 133) address this issue in lamenting that inferring relations from online data is challenging and has its pitfalls as the system is so dynamic.

Second, we had to restrict our analysis to MPs who were present in both data sets. Out of 321 MPs who served during the 48th and 49th legislative period, 163 MPs were active on Politnetz. We compared the offline, cosponsorship support among these 163 MPs with their online endorsement behavior. While we ensured that our network statistics accounts for dependencies and control-variables are calculated on the respective full networks (offline and online), we still have a likely bias due to missing observations. Additional studies with more complete comparison data sets would be valuable.

We want to stress that future studies on the external validity of online political data should avoid data sources where technical or algorithmic manipulations are at work. The freely available data on engagements of MPs on the social media site Facebook or the microblogging platform Twitter, for instance, do not provide fair assessments of the external validity of online data. Many online social media platforms provide their users with 'recommendations' of other users to follow, support, or like. These 'recommendations' possibly bias the results as users do not freely choose other users to follow, support, or like, and are presented with specific opportunities to support some users at higher rates.

Studying online political behavior can have its merits, but linking it back to real-life outcomes gives it more depth. We look forward to additional research on the topic, linking online coalition formation or online endorsement to voting outcomes or new legislative proposals by groups of MPs formed online. We examined online and offline support among MPs in a multiparty, consensual-federal democracy. Our results show that online political endorsement is guided mainly by party lines. Future studies should be conducted in different political systems and studying different political behavior on- and offline. 


\section{References}

Alvarez, Raquel, David Garcia, Yamir Moreno and Frank Schweitzer. 2015. "Sentiment cascades in the 15M movement." EPf Data Science 4(1):1-13.

Bailer, Stefanie and Sarah Bütikofer. 2015. "From loose alliances to professional political players: How Swiss party groups changed." Swiss Political Science Review 21(4):556-577.

Barberá, Pablo. 2015. "Birds of the same feather tweet together: Bayesian ideal point estimation using Twitter data." Political Analysis 23(1):76-91.

Barberá, Pablo, Andreu Casas, Jonathan Nagler, Patrick J Egan, Richard Bonneau, John T Jost and Joshua A Tucker. 2019. "Who leads? Who follows? Measuring issue attention and agenda setting by legislators and the mass public using social media data." American Political Science Review 113(4):883901.

Beauchamp, Nicholas. 2017. "Predicting and interpolating state-level polls using Twitter textual data." American fournal of Political Science 61(2):490-503.

Bond, Robert and Solomon Messing. 2015. "Quantifying social media's political space: Estimating ideology from publicly revealed preferences on Facebook." American Political Science Review 109(1):62-78.

Brandenberger, Laurence. 2018. "Trading Favors - Examining the Temporal Dynamics of Reciprocity in Congressional Collaborations Using Relational Event Models." Social Networks 54:238-253.

Brandenberger, Laurence, Giona Casiraghi, Vahan Nanumyan and Frank Schweitzer. 2019. Quantifying triadic closure in multi-edge social networks. In 2019 IEEE/ACM International Conference on Advances in Social Networks Analysis and Mining (ASONAM). IEEE pp. 307-310.

Bratton, Kathleen A and Stella M Rouse. 2011. "Networks in the legislative arena: How group dynamics affect cosponsorship." Legislative Studies Quarterly 36(3):423-460.

Burstein, Paul, Shawn Bauldry and Paul Froese. 2005. "Bill sponsorship and congressional support for policy proposals, from introduction to enactment or disappearance." Political Research Quarterly 58(2):295-302.

Burt, Ronald S and Marc Knez. 1995. "Kinds of third-party effects on trust." Rationality and society 7(3):255-292.

Campbell, James E. 1982. “Cosponsoring legislation in the US Congress." Legislative Studies Quarterly $7: 415-422$.

Casiraghi, Giona. 2017. "Multiplex Network Regression: How do relations drive interactions?" arXiv preprint arXiv:1702.02048.

Casiraghi, Giona. 2019. "The block-constrained configuration model." Applied Network Science 4(1):123.

Casiraghi, Giona and Vahan Nanumyan. 2018. "Generalised hypergeometric ensembles of random 
graphs: The configuration model as an urn problem." arXiv:1810.06495 .

Casiraghi, Giona and Vahan Nanumyan. 2020. ghypernet: Fit and Simulate Generalised Hypergeometric Ensembles of Graphs. R package version 1.0.1. https://github.com/gi0na/r-ghypernet/.

Casiraghi, Giona, Vahan Nanumyan, Ingo Scholtes and Frank Schweitzer. 2016. "Generalized Hypergeometric Ensembles: Statistical Hypothesis Testing in Complex Networks.” arXiv:1607.02441 .

Casiraghi, Giona, Vahan Nanumyan, Ingo Scholtes and Frank Schweitzer. 2017. From Relational Data to Graphs: Inferring Significant Links Using Generalized Hypergeometric Ensembles. In Social Informatics: 9th International Conference, SocInfo 2017, Oxford, UK, September 13-15, 2017, Proceedings, Part II, ed. Giovanni Luca Ciampaglia, Afra Mashhadi and Taha Yasseri. Springer International Publishing pp. 111-120.

Cherepnalkoski, Darko, Andreas Karpf, Igor Mozetič and Miha Grčar. 2016. "Cohesion and coalition formation in the European Parliament: roll-call votes and Twitter activities." PloS one 11(11):e0166586.

Cherepnalkoski, Darko and Igor Mozetič. 2016. "Retweet networks of the European Parliament: evaluation of the community structure." Applied network science 1(1):2.

Coleman, James S. 1990. Foundations of Social Theory. Cambridge, MA: Harvard University Press.

Cook, James M. 2016. "Are American politicians as partisan online as they are offline? Twitter networks in the US Senate and Maine State Legislature." Policy \& Internet 8(1):55-71.

Craig, Alison, Skyler J Cranmer, Bruce A Desmarais, Christopher J Clark and Vincent G Moscardelli. 2015. "The Role of Race, Ethnicity, and Gender in the Congressional Cosponsorship Network." arXiv preprint arXiv:1512.06141.

Cranmer, Skyler J and Bruce A Desmarais. 2011. "Inferential network analysis with exponential random graph models." Political Analysis 19(1):66-86.

Desmarais, Bruce A, Vincent G Moscardelli, Brian F Schaffner and Michael S Kowal. 2015. "Measuring legislative collaboration: The Senate press events network." Social Networks 40:43-54.

DiGrazia, Joseph, Karissa McKelvey, Johan Bollen and Fabio Rojas. 2013. "More tweets, more votes: Social media as a quantitative indicator of political behavior." PloS one 8(11):e79449.

Fischer, Manuel, Frédéric Varone, Roy Gava and Pascal Sciarini. 2019. "How MPs ties to interest groups matter for legislative co-sponsorship." Social Networks 57:34-42.

Fowler, James H. 2006. "Connecting the Congress: A study of cosponsorship networks." Political Analysis 14(4):456-487.

Friedkin, Noah E. 2004. "Social cohesion." Annu. Rev. Sociol. 30:409-425.

Garcia, David, Adiya Abisheva, Simon Schweighofer, Uwe Serdült and Frank Schweitzer. 2015. "Ideological and temporal components of network polarization in online political participatory media." Policy \& internet 7(1):46-79. 
Garcia, David, Fernando Mendez, Uwe Serdült and Frank Schweitzer. 2012. Political polarization and popularity in online participatory media: an integrated approach. In Proceedings of the first edition workshop on Politics, elections and data-PLEAD'12. ACM pp. 3-10.

Granovetter, Mark. 1985. "Economic action and social structure: The problem of embeddedness." American journal of sociology 91(3):481-510.

Granovetter, Mark S. 1973. "The strength of weak ties." American journal of sociology 78(6):1360-1380.

Grimmer, Justin. 2015. "We are all social scientists now: How big data, machine learning, and causal inference work together." PS, Political Science \& Politics 48(1):80.

Kessler, Daniel and Keith Krehbiel. 1996. "Dynamics of cosponsorship." American Political Science Review 90(03):555-566.

Kirkland, Justin H. 2011. "The relational determinants of legislative outcomes: Strong and weak ties between legislators." The fournal of Politics 73(3):887-898.

Kirkland, Justin H and Justin H Gross. 2014. "Measurement and theory in legislative networks: The evolving topology of Congressional collaboration." Social Networks 36:97-109.

Krehbiel, Keith. 1995. "Cosponsors and Wafflers from A to Z." American fournal of Political Science 39(4):906.

Mayhew, David R. 1974. Congress: The electoral connection. New Haven, CT: Yale University Press.

Melamed, David and Brent Simpson. 2016. "Strong ties promote the evolution of cooperation in dynamic networks." Social Networks 45:32-44.

Peoples, Clayton D. 2008. "Interlegislator Relations and Policy Making: A Sociological Study of Roll-Call Voting in a State Legislature 1." Sociological Forum 23(3):455-480.

R Core Team. 2020. R: A Language and Environment for Statistical Computing. Vienna, Austria: R Foundation for Statistical Computing. https://wWw.R-project.org/ (last access: June 2020).

Schiller, Wendy J. 1995. "Senators as political entrepreneurs: Using bill sponsorship to shape legislative agendas." American fournal of Political Science pp. 186-203.

Schwarz, Daniel. 2009. Zwischen Fraktionszwand und freiem Mandat. Eine Untersuchung des fraktionsabweichenden Stimmverhaltens im schweizerischen Nationalrat zwischen 1996-2005 Doktorwürde Universität Bern Bern: .

Simmel, Georg. 1908 (1950). The Sociology of Georg Simmel. New York: Free Press.

Spector, Paul E and Michael T Brannick. 2011. "Methodological urban legends: The misuse of statistical control variables." Organizational Research Methods 14(2):287-305.

Talbert, Jeffery $\mathrm{C}$ and Matthew Potoski. 2002. "Setting the legislative agenda: The dimensional structure of bill cosponsoring and floor voting." The fournal of Politics 64(03):864-891. 
Tam Cho, Wendy K and James H Fowler. 2010. "Legislative success in a small world: Social network analysis and the dynamics of congressional legislation." The fournal of Politics 72(01):124-135.

Weaver, Iain S, Hywel Williams, Iulia Cioroianu, Matthew Williams, Travis Coan and Susan Banducci. 2018. "Dynamic social media affiliations among UK politicians." Social networks 54:132-144.

Wilson, Rick K and Cheryl D Young. 1997. "Cosponsorship in the US Congress." Legislative Studies Quarterly 22:25-43.

Woon, Jonathan. 2008. "Bill sponsorship in Congress: the moderating effect of agenda positions on legislative proposals." The fournal of Politics 70(1):201-216. 